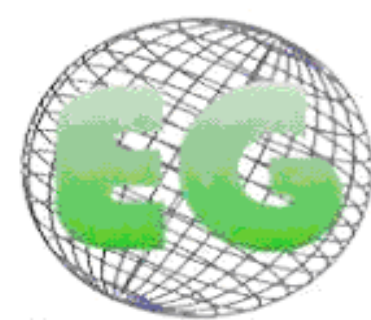

ISSN 1695-6141 $\mathbf{N}^{\circ} 22$
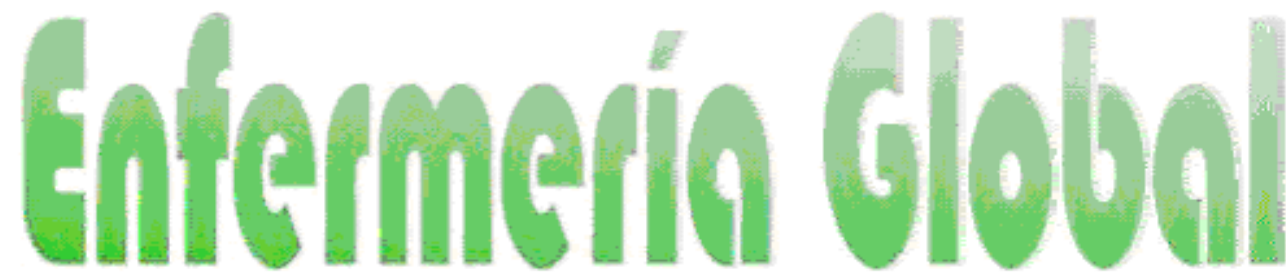

Revista electrónica trimestral de Enfermería

Abril 2011

\title{
EL FENÓMENO DE LA VIOLENCIA DE GÉNERO EN LA MUJER A PARTIR DE LA PRODUCCIÓN CIENTIIFICA DE ENFERMERÍA
}

O FENÔMENO DA VIOLÊNCIA DE GENERO À MULHER A PARTIR DA PRODUÇÃO CIENTÍIFICA DA ENFERMAGEM

*Rangel da Silva, L., "*Domingues Bernardes Silva, M., ${ }^{* * *}$ Mota Xavier de Meneses, T., " ${ }^{* * * * R o d r i ́ g u e z ~ B o r r e g o, ~ M A ., ~ " M e n e s e s ~ d o s ~ S a n t o s, ~ I M ., ~ * * * * * L e m o s, ~}$ A.

*Doctora en Enfermería. Profesora Adjunta del Departamento Materno-Infantil de la Escuela de Enfermería Alfredo Pinto. Universidad Federal del Estado de Rio de Janeiro (UNIRIO). Coordinadora del Núcleo de Pesquisa, Estudos e Experimentação em Enfermagem na Área da Mulher e da Criança (NuPEEMC). **Enfermera. Miembro del NuPEEMC . ${ }^{* * * B}$ Bolsista de Iniciación Científica de UNIRIO. Graduanda de la EEAP. Miembro del NuPEEMC. ***EEnfermera Doctora. Profesora Titular de Escuela Universitaria. Universidad de Córdoba (España). ${ }^{* * * * E n f e r m e r a . ~ D o c t o r a ~ e n ~ S a l u d ~ C o m u n i t a r i a . ~ A d j u n t a ~ d e l ~ D e p a r t a m e n t o ~ d e ~ E n f e r m e r i ́ a ~}$ de Salud Pública de la EEAP/UNIRIO.Brasil.

(Este artículo forma parte del proyecto financiado por el CNPq: " Violência de Gênero à Mulher Enfermeira" bajo la coordinación de la Profa Dra. Leila Rangel da Silva, e integra Grupos de Pesquisa de España y Portugal, del Proyecto Internacional de mismo título. La investigación en España ha sido financiada por la Junta de Andalucía. № expediente: PI-0109/2008).

Palabras clave: Enfermería, Violencia contra la Mujer, Género y Salud.
Palavras-chave: Enfermagem; Violência contra a mulher; Gênero e Saúde.

Keywords: Nursing; Violence against Women; Gender and Health

\section{RESUMEN}

Con el objetivo de identificar y analizar la producción científica de Enfermería sobre la violencia contra la mujer en el período 2000 a 2009, se realizó una revisión de la literatura científica en el portal de revistas de enfermería de la Biblioteca Virtual en Salud de Brasil. A partir de los criterios de inclusión, fueron identificadas 10 publicaciones que describían explícitamente la relación de la violencia de género y sus implicaciones en el cuidado de la mujer. A partir del análisis temático de los artículos se obtuvieron cuatro categorías: intentar cuantificar y explicar el fenómeno de la violencia; el fenómeno relacionado con el profesional de la salud; el aprendizaje de la violencia, y la 
relación con el compañero. El conocimiento obtenido sobre el tema en estudio da una visión desde la perspectiva de la enfermera, sin embargo, no aborda de lleno la perspectiva del cuidado, lo que estimula a la producción de otros estudios sobre este tema.

\section{RESUMO}

Com o objetivo de identificar e analisar a produção científica na área da enfermagem sobre violência contra a mulher no período de 2000 a 2009, realizou-se uma revisão bibliográfica da produção científica consultando o portal de revistas de enfermagem da Biblioteca Virtual em Saúde do Brasil. A partir dos critérios de inclusão, foram identificadas 10 publicações que descreviam explicitamente a relação da violência de gênero e suas implicações no cuidado à mulher. A partir da análise temática, emergiram quatro categorias: tentativa de quantificar e explicar o fenômeno da violência; fenômeno relacionado ao profissional de saúde; a aprendizagem da violência e a relação com o companheiro. O conhecimento obtido oferece uma visão desde a perspectiva da enfermeira sobre o tema em estudo, entretanto não aborda por completo a perspectiva do cuidado, o que estimula a produção de outros estudos nesta temática.

\section{ABSTRACT}

In order to identify and analyze the scientific production in the area of nursing on violence against women in the period from 2000 to 2009 , a study was made of scientific literature in which the first page of nursing journals of the Virtual Health Library of Brazil was consulted. Based on the criteria of inclusion, 10 publications that explicitly described the relationship of gender violence and its implications in w omen's care were identified. On considering the thematic analysis, four categories emerged: attempts to quantify and explain the phenomenon of violence; phenomenon related to the health professional; the learning of violence and the relationship with a partner. The knowledge acquired offers a view from nurse's perspective on the analyzed topic; however, the study does not manage to outline a complete perspective of care, which stimulates the production of other studies on this subject.

\section{INTRODUCCIÓN}

El objeto de este estudio es el fenómeno de la violencia de género contra la mujer a partir de la producción científica de enfermería. Se trata de un subproyecto del Proyecto de Investigación "Violencia de Género en la Mujer Enfermera" financiado por el CNPq y cadastrado en el Núcleo de Pesquisa, Estudos e Experimentação na Área da Saúde da Mulher e da Criança (NuPEEMC) del Departamento de Enfermería Materno-Infantil (DEMI) de la Universidad Federal del Estado de Rio de Janeiro (UNIRIO) y vinculado al Proyecto "Cuidado Cultural a la Salud de la Mujer Brasileña-Tendencias y Desafíos para la Enfermería" bajo la responsabilidad de la Prof ${ }^{a}$ Dr $^{a}$. Leila Rangel da Silva.

El vocablo violencia viene de la palabra latina vis, que quiere decir fuerza, y se refiere a las nociones de restricción y de uso de la superioridad física sobre el otro. La violencia es un fenómeno extremadamente difuso y complejo cuya definición no puede tener exactitud científica, ya que es una cuestión de apreciación y está influida por la cultura y sometida a una continua revisión en la medida en que los valores y las normas sociales evolucionan ${ }^{(1)}$.

De esta forma, percibimos que el fenómeno de la violencia es cambiante, pues sufre influencias históricas, geográficas, circunstanciales y de realidades muy diferentes. Existen violencias toleradas y condenadas, ya que la violencia existe desde que el hombre vive sobre la Tierra, presentándose bajo diferentes formas, cada vez más complejas y al mismo tiempo más fragmentadas y articuladas ${ }^{(2)}$. De esta forma hay una variedad de definiciones 
de la violencia de género, en un intento de conceptualizar el fenómeno y proporcionar una dirección única para quien precisa trabajar con él ${ }^{(3)}$.

Durante todas las fases de nuestra vida sufrimos influencias de instituciones como la escuela, la iglesia, el trabajo y en la propia familia, con lo que desde el principio aprendemos determinadas conductas, en última instancia, a representar los papeles atribuidos a los géneros, lo que influirá en la construcción de la identidad de género.

En lo que atañe a las denominaciones violencia doméstica y violencia familiar, la Ley Maria da Penha, en su Art. 5으, hace referencia a la violencia conyugal como aquella que se da "en cualquier relación íntima de afecto, en la que el agresor conviva o haya convivido con la ofendida, independientemente de cohabitación" (4). La violencia dirigida contra la mujer consiste en todo acto de violencia de género que resulte en cualquier acción física, sexual o psicológica, incluida la amenaza ${ }^{(5)}$.

Para entender la denominación de violencia de género es preciso considerar el carácter social de las trazas atribuidas a hombres y mujeres. De esta forma, se observa que la mayoría de las trazas de lo femenino y de lo masculino son construcciones culturales, son productos de la sociedad y no derivados necesariamente de la naturaleza ${ }^{(6)}$. La determinación en ser hombre o ser mujer es social e histórica y no biológica.

En el modelo de familia tradicional, los atributos y los roles de género valoran al hombre en detrimento de la mujer, legitimando, por un lado, la dominación del hombre y por otro, la inferioridad de la mujer. En esta perspectiva, la mujer es destituida de autonomía y del derecho a decidir, inclusive sobre su propio cuerpo ${ }^{(7)}$.

En todas las culturas del mundo, las mujeres viven en condiciones de desigualdad social en relación a los hombres. Hay una significativa repercusión en la salud física y mental de los individuos que presencian o sufren las diversas formas de violencia en su cotidiano. Para minimizar sus consecuentes agravios, es necesaria una atención especial, juiciosa y profunda dirigida a la atención de la salud con un enfoque holístico y contextualizado.

Diversas conferencias internacionales realizadas en el siglo XX contienen los enunciados y las definiciones de los derechos humanos para todos los habitantes del mundo, los cuales, sin duda, ayudaron mucho en la detección e investigación de la violencia de género en la mujer. Estos convenios fueron: Carta de las Naciones Unidas (1945); Convenio contra el genocidio (1948); Pacto internacional de los derechos civiles y políticos (1966); Pacto internacional de los derechos económicos, sociales y culturales (1966); Convenio para la eliminación de todas las formas de discriminación contra la mujer (1979); Convenio sobre los derechos del niño (1989); y Convenio interamericano para prevenir, punir y erradicar la violencia contra la mujer - Convenio de Belém do Pará (1994) ${ }^{(8)}$. En 1995 la Conferencia Mundial sobre la Mujer, en Beijing en su Plataforma de Acción dedicó todo un capítulo a la eliminación de la violencia contra las mujeres.

La relación entre la violencia contra la mujer y su salud se hace cada vez más evidente, aunque la mayoría de las mujeres no relata que sufre la violencia doméstica. Por eso, es extremadamente importante que los profesionales de salud estén capacitados para identificar, atender y tratar a las mujeres que presentan síntomas que pueden estar relacionados con el abuso sexual y la agresión física.

Frente a la necesidad de percibir y comprender el fenómeno de la violencia contra la mujer, es necesario hacer análisis y estudios sobre el asunto, pues tal violencia deteriora la salud 
individual y familiar de la mujer. Este estudio surge en la tentativa de proporcionar mayor visibilidad a los profesionales de enfermería acerca de la producción científica producida y publicada con la temática de violencia de género contra la mujer.

Para el desarrollo del estudio se trazaron los siguientes objetivos: Identificar y analizar la producción científica en el área de enfermería sobre violencia contra la mujer en el periodo de 2000 a 2009.

A partir de esto, la justificativa de este estudio se respalda en la necesidad de conocer sobre el conocimiento producido acerca de la violencia de género a la mujer, y con esto, proporcionar a los profesionales de salud un panorama sobre el tema, para que puedan ser valorados los principales cuidados con esta mujer que presencia o sufre el fenómeno de la violencia.

\section{ABORDAJE METODOLÓGICO}

Para alcanzar los objetivos de este estudio, elegimos los principios de la investigación bibliográfica, que comprende la lectura, selección, informe y archivo de los tópicos de interés para la investigación en pauta, para conocer las contribuciones científicas que se efectuaron sobre determinado asunto ${ }^{(9)}$.

Para esta revisión bibliográfica se adoptó como criterio de inclusión: artículos que versan sobre la temática violencia de género contra la mujer; producciones que describen explícitamente la relación de la violencia de género a la mujer y sus implicaciones en el cuidado a la mujer; el periodo de las publicaciones de 2000 a 2009 y tener en los descriptores, las palabras: Violencia y/o Violencia contra la Mujer y/o Identidad de Género y/o Género y Salud.

En un primer momento de nuestra investigación consultamos SciELO, a través del sitio http://www.scielo.br, y a partir de ahí se realizó una busca en el portal de revistas de enfermería (REVeENF) de la Biblioteca Virtual em Saúde (BVS). Utilizando el descriptor violencia, se encontraron 109 artículos. Tras ese momento, se seleccionaron artículos que versaban específicamente sobre la temática de la violencia de género o la violencia contra la mujer y de ese total, solo 12 artículos fueron identificados.

En un segundo momento, se utilizaron todos los criterios de inclusión arriba citados. De esta manera, se seleccionaron 10 producciones científicas sometiéndolas a la siguiente clasificación: año y lugar de publicación, revista científica, metodología empleada y la idea central.

Finalmente, se empleó la técnica de análisis temático. Esa técnica "consiste en descubrir los núcleos de sentido que componen la comunicación y cuya presencia puede significar alguna cosa para el objetivo analítico escogido" ${ }^{(10)}$.

\section{RESULTADOS Y DISCUSIÓN DE LOS DATOS}

De las 10 producciones bibliográficas publicadas entre 2004 y 2009 , una es de origen internacional, de Chile ${ }^{(11)}$ y nueve son de origen nacional, siendo siete de la región sudeste (12-13-14-15-16-17-18), una de la región centro oeste ${ }^{(19)}$ y una de la región sur ${ }^{(20)}$. 
Vale resaltar que las producciones científicas identificadas en el portal de revistas de enfermería a partir de los criterios de inclusión delimitados para ese estudio, fueron publicados a partir de 2004.

La mayor cantidad de producción nacional puede justificarse a partir de la creación de políticas públicas de salud dirigidas a la humanización de la asistencia a la mujer. Internacionalmente, destaca el Relatório Mundial sobre Violência e Saúde, una publicación histórica hecha por la Organización Mundial de la Salud (OMS), donde traza visibilidad a este tema, así como también ayuda a investigadores y profesionales sobre posibles acciones de promoción de la salud, prevención y diagnóstico de la violencia. Además, esta organización internacional revela la magnitud de este problema, señalando la violencia contra la mujer como una de las cinco prioridades para las Américas en los años iniciales del siglo XXI ${ }^{(1)}$. Los artículos científicos seleccionados para el estudio están descritos en el Cuadro I.

Cuadro I- Relación de los artículos encontrados: autor, título, año de publicación y revista.

\begin{tabular}{|c|c|c|c|}
\hline Autor & Título & $\begin{array}{l}\text { Año de } \\
\text { publicación }\end{array}$ & Revista \\
\hline $\begin{array}{l}\text { Okabe I, Fonseca } \\
\text { RMGS da }\end{array}$ & $\begin{array}{ll}\text { Violência contra a } \\
\text { mulher: contribuições } \\
\text { e limitações do } \\
\text { sistema } & \text { de } \\
\text { informação } & \\
\end{array}$ & 2009 & $\begin{array}{l}\text { Revista da } \\
\text { Escola de } \\
\text { Enfermagem } \\
\text { da USP }\end{array}$ \\
\hline $\begin{array}{lll}\text { Trindade } & \text { RFC da, } \\
\text { Almeida AM de, } \\
\text { Rozendo CA }\end{array}$ & $\begin{array}{l}\text { Infidelidade } \\
\text { masculina e violência } \\
\text { doméstica: vivência } \\
\text { de um grupo de } \\
\text { mulheres }\end{array}$ & 2008 & $\begin{array}{l}\text { Ciencia y } \\
\text { Enfermería }\end{array}$ \\
\hline $\begin{array}{l}\text { Andrade CJM, } \\
\text { Fonseca RMGS da }\end{array}$ & $\begin{array}{l}\text { Considerações sobre } \\
\text { violência doméstica, } \\
\text { gênero e o trabalho } \\
\text { das equipes de } \\
\text { saúde da família }\end{array}$ & 2008 & $\begin{array}{l}\text { Revista da } \\
\text { Escola de } \\
\text { Enfermagem } \\
\text { da USP }\end{array}$ \\
\hline $\begin{array}{l}\text { Lettiere A, Nakano } \\
\text { AMS, Rodrigues DT }\end{array}$ & $\begin{array}{lr}\text { Violência contra a } \\
\text { mulher: a visibilidade } \\
\text { do problema para um } \\
\text { grupo } \\
\text { profissionais de } \\
\text { saúde }\end{array}$ & 2008 & $\begin{array}{l}\text { Revista da } \\
\text { Escola de } \\
\text { Enfermagem } \\
\text { da USP }\end{array}$ \\
\hline $\begin{array}{ll}\text { Monteiro } & \text { CFS, } \\
\text { Souza IEO } & \end{array}$ & $\begin{array}{l}\text { Vivência da violência } \\
\text { conjugal: fatos do } \\
\text { cotidiano }\end{array}$ & 2007 & $\begin{array}{l}\text { Texto } \\
\text { Contexto de } \\
\text { Enfermagem }\end{array}$ \\
\hline $\begin{array}{l}\text { Gomes NP, Diniz } \\
\text { NMF, Araújo AJS, } \\
\text { Coelho TMF }\end{array}$ & $\begin{array}{l}\text { Compreendendo a } \\
\text { violência doméstica } \\
\text { a partir das } \\
\text { categorias gênero e } \\
\text { geração }\end{array}$ & 2007 & $\begin{array}{l}\text { Acta } \\
\text { Paulista de } \\
\text { Enfermagem }\end{array}$ \\
\hline
\end{tabular}




\begin{tabular}{|c|c|c|c|}
\hline $\begin{array}{l}\text { Guedes RN, Silva } \\
\text { ATMC da, Coelho } \\
\text { EAC }\end{array}$ & $\begin{array}{l}\text { Violência conjugal: } \\
\text { problematizando a } \\
\text { opressão das } \\
\text { mulheres vitimizadas } \\
\text { sob o olhar de } \\
\text { gênero }\end{array}$ & 2007 & $\begin{array}{l}\text { Revista } \\
\text { Eletrônica } \\
\text { de } \\
\text { Enfermagem }\end{array}$ \\
\hline $\begin{array}{ll}\text { Casique } & \mathrm{LC}, \\
\text { Furegato ARF } & \end{array}$ & $\begin{array}{lr}\text { Violência } & \text { contra } \\
\text { mulheres: } & \text { reflexões } \\
\text { teóricas } & \end{array}$ & 2006 & $\begin{array}{l}\text { Revista } \\
\text { Latino- } \\
\text { Americana } \\
\text { de } \\
\text { Enfermagem }\end{array}$ \\
\hline $\begin{array}{ll}\text { Araújo } & \text { LM, } \\
\text { Progianti } & \text { JM, } \\
\text { Vargens OMC } & \end{array}$ & $\begin{array}{l}\text { A consulta de } \\
\text { enfermagem } \\
\text { ginecológica e a } \\
\text { redução da violência } \\
\text { de gênero }\end{array}$ & 2004 & $\begin{array}{l}\text { Revista de } \\
\text { Enfermagem } \\
\text { da UERJ }\end{array}$ \\
\hline $\begin{array}{l}\text { Almeida LCG, Diniz } \\
\text { NMF }\end{array}$ & $\begin{array}{lr}\text { Violência } & \text { sexual: } \\
\text { desvelando } & \text { a } \\
\text { realidade } & \text { que } \\
\text { acomete } & \text { as } \\
\text { mulheres } & \\
\end{array}$ & 2004 & $\begin{array}{l}\text { Revista de } \\
\text { Enfermagem } \\
\text { da UERJ }\end{array}$ \\
\hline
\end{tabular}

En cuanto a la metodología empleada en los artículos seleccionados, tenemos cuatro revisiones bibliográficas $^{(12-13-14-15)}$, una revisión de literatura ${ }^{(16)}$ y cinco pesquisas cualitativas, de las que cuatro utilizaron entrevistas como técnica de colecta de datos ${ }^{(11,17,19-20)}$ y una, relato de experiencia ${ }^{(18)}$

En lo tocante a la idea central trazada por los artículos, la temática de la violencia de género fue abordada en un artículo ${ }^{(15)}$ por el aspecto del sistema de información que cuantifica el fenómeno. En tres artículos ${ }^{(13,17-18)}$ fueron correlacionados aspectos de la violencia doméstica con el profesional de salud. En dos artículos ${ }^{(19-20)}$ se centró la violencia conyugal. Solo una producción abordó la violencia sexual ${ }^{(12)}$. Un estudio ${ }^{(14)}$ hizo un breve histórico de la violencia de género contra la mujer y sus consecuencias para la salud, afirmando ser necesario el uso de un modelo ecológico para el estudio de ese fenómeno, pues este contempla la complejidad del fenómeno. En una investigación ${ }^{(11)}$ se abordó la cuestión de la infidelidad masculina y la violencia de género. Hay un artículo ${ }^{(16)}$ que abordó la violencia de género y la violencia intergeneracional. En el periodo estudiado y en los lugares investigados, predominó la producción implicando al profesional de salud.

De esta forma, tras la selección del material de estudio, se utilizó el análisis de contenido en la modalidad temática que trata las informaciones colectadas sobre un determinado asunto resultado en la clasificación de temas ${ }^{(10)}$. A partir del análisis temático, aparecieron cuatro categorías, a saber: 1) Tentativa de cuantificar y explicar el fenómeno de la violencia; 2) Fenómeno relacionado con el profesional de salud; 3) El aprendizaje de la violencia y 4) La relación con el compañero.

\section{I - Tentativa de cuantificar y explicar el fenómeno de la violencia}

Los inconvenientes y las ventajas de la cuantificación y del sistema de información sobre la violencia, permiten comparaciones entre las regiones del país con otros lugares por la estandarización internacional de la información y visualización más concreta del fenómeno, evaluando su amplitud y su impacto ${ }^{(15)}$. 
El sistema de información en salud, a pesar de ser el principal banco de datos para el seguimiento de las causas externas, posee algunos factores que limitan el dimensionamiento de la violencia contra la mujer en Brasil, como el rellenar los datos según la morbilidad y la mortalidad en registros separados, lo que dificulta el análisis conjunto de la morbimortalidad de esas causas; la poca sensibilidad y representatividad del acontecimiento debido al subregistro de los casos por la probable razón de ser reciente el reconocimiento de la violencia como un problema de salud colectiva y la falta de capacitación del profesional de salud para luchar contra el fenómeno de la violencia, además de la concepción del sistema de información utilizado actualmente, que no fue configurado para esta finalidad, asociado a la falta de uniformidad en el modo como los datos son colectados, lo que hace más difícil la comparación entre las diferentes realidades ${ }^{(15)}$.

La comprensión socio-histórica y también cultural del proceso expone los varios tipos de violencia y la forma como cada uno se expresa, situando también las consecuencias sobre la salud de la mujer. El modelo ecológico, que valora algunas de las relaciones que corresponden al individuo y su entorno, daría cuenta de un fenómeno tan complejo ${ }^{(14)}$.

\section{II- Fenómeno relacionado con el profesional de salud}

En los artículos analizados en esta categoría, encontramos una producción que valora la utilización de estrategias para reducir la violencia de género contra la mujer en la consulta de enfermería ginecológica como: la escucha sensible, la participación de una persona de confianza de esta mujer en la consulta, no prohibir el uso de la sábana sobre la cintura y el incentivo a la mujer de introducir el espéculo vaginal en ella. Por tanto, el enfermero debe reflexionar sobre su práctica asistencial, para no ser un agente reproductor de la violencia (18).

El sentimiento de la violencia por los profesionales de salud es un problema serio e importante en la sociedad y se manifiesta de varias formas. Los profesionales remiten a una causa multifactorial para el fenómeno, sin embargo, reconocen ser causa y consecuencia de las desigualdades de género anclada en la construcción de la identidad de cada género. Hay valorización de las quejas físicas, el cuidado centrado en los aspectos biológicos, prevaleciendo la relación técnica instrumental del cuidado. También hay falta de orientación en la graduación para luchar con el tema y cuando este se presenta, es fragmentado. Hay relatos de profesionales de salud en cuanto a las sensaciones de incomodidad e impotencia, miedo a ser ofensivos, pérdida del control de la situación y restricción ${ }^{(17)}$.

A pesar de las dificultades de los profesionales en atender a las mujeres en las demandas de este fenómeno, está la necesidad de profesionales del equipo de salud de familia para "discutir la cuestión de la violencia doméstica en la cotidianidad de los servicios de salud capacitando profesionales y asociándose con otros servicios" reorganizando las prácticas asistenciales ${ }^{(13)}$.

\section{III- El aprendizaje de la violencia}

La violencia de género se va construyendo a partir de los papeles atribuidos a niñas/niños, hombre/mujer dentro de la familia, fruto de la cultura patriarcal. Dentro de la familia, las relaciones tienden a valorar al hombre en detrimento de la mujer. En la violencia intergeneracional se reproduce su historia vivida en la infancia o adolescencia. El núcleo familiar es, por tanto, un espacio de construcción y reproducción de la violencia doméstica (16). 


\section{IV- La relación con el compañero}

La violencia conyugal fue estudiada buscando su significado. Encontraron en las palabras de las mujeres la vivencia del fenómeno a través de síntomas de orden físico, a través de un cotidiano inmerso en conflictos constantes con la pareja, descritos por violencia física, sexual y psicológica y a través de carencia de cuidados y afecto, encarcelamiento, aislamiento, baja autoestima. A través de estos resultados se ve que no siempre las evidencias de la violencia son visibles, son marcas de la dimensión subjetiva y solo pueden ser conocidas cuando se expresan de palabra ${ }^{(20)}$.

Hubo interés por el significado de la violencia conyugal en otra pesquisa. El artículo presenta una categoría que sintetiza el sentido de las palabras de las mujeres: La violencia conyugal y su relación con la tutela de la diferencia en las relaciones desiguales de género: la asimetría del poder impuesto a la sumisión y el silencio de las mujeres en situación de violencia. Esta idea muestra que el desequilibrio de poder a favor del hombre caracteriza la desigualdad de género, ello denota privilegio y es contrario al principio democrático de igualdad. Con todo, existen formas sociales de reproducción de la violencia a través de la familia que naturalizan el fenómeno ${ }^{(19)}$.

El contrato de casamiento es entendido como una relación de trabajo, pues a cambio de sustento y protección, el marido recibe el trabajo doméstico y el acceso sexual al cuerpo de la esposa, por lo que el casamiento no se muestra en las mujeres oídas como un disfrute sexual igualitario entre ambos ${ }^{(19)}$.

La mujer estuvo, por mucho tiempo, excluida del espacio público, limitadas sus actividades solo al hogar, sin autonomía e independencia financiera. Se nota, por tanto, que al ingresar en el mercado de trabajo no quedaron excluidas de la responsabilidad del hogar lo que causa sobrecarga y sobre todo un factor de opresión. A pesar de ingresar en el mercado de trabajho, pocas mujeres tienen puestos bien remunerados, recibiendo menores salarios que los hombres en el mismo cargo, siendo, muchas veces, dirigidas por ellos, lo que indica que el mercado es patriarcal ${ }^{(19)}$. Este estudio reveló que en el espacio público del mercado de trabajo y en el espacio privado de las relaciones familiares, las relaciones de género intensifican las desigualdades asociadas a las clases sociales. A pesar de que la independencia financiera y del acceso al mercado de trabajo ofrecen mejores condiciones para la conquista de la autonomía y libertad, no son condición suficiente para posibilitar a las mujeres relaciones de género más igualitarias ${ }^{(19)}$.

La violencia sexual fue discutida con sus repercusiones en la salud de la mujer ${ }^{(12)}$. El texto aborda que género es una forma de significar relaciones de poder, es un elemento constitutivo de relaciones sociales basado en las diferencias percibidas entre los sexos. La violencia se aprende socialmente y se establece en relaciones asimétricas de poder. Algunos mitos incluyen la mujer víctima de abuso sexual, por ejemplo: que los policías no acreditan la veracidad de las historias de las víctimas, no acreditan la veracidad de los sentimientos de impotencia y las manifestaciones emocionales presentadas son entendidas como síntomas de patologías ${ }^{(12)}$.

También hay una justificativa de la violencia como el mito de que solo las mujeres promiscuas son violadas. La cuestión de la violencia va ganando en importancia en el área da salud, toda vez que el hospital y el puesto de salud pueden ser la puerta de entrada de estas mujeres, necesitando que el profesional de salud conozca que este fenómeno no siempre deja marcas visibles ${ }^{(12)}$. La violencia sexual puede estar admitida dentro del propio 
hogar y esto desnaturaliza la obligación sexual solo para la mujer y puede representar avances en el combate contra la sumisión femenina. En cuanto a los riesgos para la salud existe una mayor vulnerabilidad a otras formas de violencia, a trastornos psicológicos, a afecciones ginecológicas y del tracto genitourinario, a heridas, a infecciones sexualmente transmisibles, a embarazo y traumatismos, entre otros ${ }^{(12)}$.

La infidelidad masculina y la relación vivida con las parejas fuerom estudiadas desde la óptica de la violencia de gênero ${ }^{(11)}$. 'Las mujeres del estudio se sienten traicionadas, agredidas y sin libertad, viéndose imposibilitadas de rehacer y modificar la situación. Las parejas de las mujeres estudiadas ejercen un patrón más autónomo en relación a su sexualidad y la mujer está subordinada a esa actitud de la naturaleza masculina. Incluso subordinadas a ese patrón, en su narrativa muestran la imposibilidad de actuar a causa de la dependencia económica.

Sobre la interpretación de la violencia, hubo dualidad en la no aceptación de la agresión procurando vengarla y la relación de la agresión con el alcohol. La violencia, a la que las mujeres del estudio están sometidas, está relacionada con el uso abusivo del alcohol y la traición que están, socialmente, permitidos al hombre y contra los cuales las mujeres no pueden luchar ${ }^{(11)}$.

La violencia entre las parejas se caracterizó con lesiones corporales, sin haber denuncia, lo que demuestra el predomino de asimetrías de género por la pasividad en que se encuentran. Las uniones del estudio surgieron para dar aceptabilidad social a los embarazos no planeados. Tales uniones con el tiempo fueron cuestionadas por las parejas en cuanto al fin de su libertad y la carga de responsabilidades para la supervivencia de la familia. La infidelidad y la forma encontrada para huir de esas responsabilidades. En algunos casos, la solución encontrada fue la separación, tomada por la mujer, mostrando su capacidad resolutiva. Separación y reconciliación también fueron vividas por el motivo de la permanencia de contacto de los padres con los hijos, lo que puede estar enmascarando la dependencia que ellas tienen del compañero. Las mujeres en el caso de la separación a veces incluso se culpabilizaron del fracaso de la relación afirmando no haber tenido competencia para manter la relación ${ }^{(11)}$.

\section{CONSIDERACIONES FINALES}

Los estudios analizados presentaron la violencia como un fenómeno complejo, invisible y naturalizado por las formas de socialización insertas en nuestra cultura. Muchas de las cuestiones involucradas limitan la autonomía de esas mujeres, sin embargo, muchas mujeres se mostraron capaces de rehacer ese camino. Es importante considerar que muchas de las expresiones de violencia sufridas surgen como un daño físico, y el sistema de salud se muestra como una puerta de entrada importante para la primera atención de esas mujeres. Debe haber en ese medio profesionales de salud, en especial de enfermería, capaces de ofrecer una acogida adecuada y de ofrecer oportunidades de expresión de sentimientos, además de realizar el camino apropiado.

Es importante la iniciativa de políticas públicas de salud para la lucha contra la violencia contra la mujer, la inserción de la temática violencia en las instituciones de enseñanza, la disponibilidad del profesional para la acogida como iniciativas importantes para restablecer la salud de personas víctimas de violencia.

Otro camino importante, según las producciones científicas de enfermería, en el intento de romper la propagación social de la violencia consiste en reestructurar la familia procurando 
construir relaciones más simétricas entre hombres y mujeres, modificando a continuación el comportamiento social anclado en la violencia de género. Se hace necesario también romper el ciclo de desigualdad social y de género en que esas mujeres están insertadas.

Sin duda, los caminos para intentar construir una sociedad más igualitaria entre hombres y mujeres y que con eso no se admita más la violencia de género, reconociéndola y combatiéndola son diversos. Por tanto, el nivel relacional de contacto con la persona violada será siempre el más transformador, pues permitirá al sujeto extrañarse de su situación o la posibilidad de nuevas opciones.

\section{REFERENCIAS}

1. Organización Panamericana de la Salud, Oficina Regional para las Américas. Informe mundial sobre la violencia y la salud. Washington: OMS; 2002.

2. Minayo SMC, Souza RE, organizadoras. Violência sob o olhar da saúde: a infrapolítica da contemporaneidade brasileira. Rio de Janeiro (RJ): Fiocruz; 2003.

3. Stein Backes D, Petters GregórioVR, Kletemberg G, Costa E, Arzuaga MA, Ramos Machado R, Pires de Pires DE. O ser humano e a perspectiva de gênero influenciando o viver e a saúde. Enfermería Global, out 2008; 14: 1-11

4. Brasil. Leis, etc. Lei n. 11.340, de 7 de agosto de 2006. Dispõe sobre a criação dos juizados de violência doméstica e familiar contra a mulher e dá outras providências. Diário Oficial da União, Brasília (DF) 2006. p.1.

5. Brasil. Ministério da Saúde. DATASUS. Conferência Nacional de Saúde. A violência contra a mulher é também uma questão de saúde pública [texto na Internet]. Belo Horizonte, 25 de novembro de 1998. [citado 2009 Fev 15] Disponível em: http://www.datasus.gov.br/cns/temas/tribuna/violencia contra mulher.htm

6. Alberdi I, Matas N. La violencia doméstica: informe sobre los malos tratos a mujeres en España. Barcelona (ES): Fundación "La Caixa"; 2002.

7. Crepschi JLB. Significações psicológicas dadas à violência sexual por mulheres atendidas em ambulatório especializado universitário: um estudo clínico-qualitativo [tese]. Campinas: Faculdade de Ciências Médicas da Universidade Estadual de Campinas, 2005.

8. Schraiber LB, D'Oliveira AFPL. O que devem saber os profissionais de saúde para promover os direitos e a saúde das mulheres em situação de violência doméstica. Projeto Gênero, violência e Direitos Humanos - Novas Questões para o Campo da Saúde. $2^{\underline{a}}$ ed. São Paulo: Fundação Ford, CREMESP; 2003.

9. Ferrari AT. Metodologia da pesquisa científica. São Paulo (SP): McGraw-Hill do Brasil; 1982.

10. Bardin L. Análise de Conteúdo. Lisboa: Edições 70; 2008.

11. Trindade RFC, Almeida AM, Rozendo CA. Infidelidade masculina e violência doméstica:vivência de um grupo de mulheres. Ciencia y Enfermeria, 2008; 14(2): 39-46.

12. Almeida LCG, Diniz NMF. Violência sexual: desvelando a realidade que acomete as mulheres. Rev Enferm UERJ 2004; 12: 88-94

13. Andrade CJM, Fonseca RMGS. Considerações sobre violência doméstica, gênero e o trabalho das equipes de saúde da família. Rev Esc Enferm USP 2008; 42(3): 591-5.

14. Casique LC, Furegato ARF. Violência contra mulheres: reflexões teóricas. Rev Latinoam Enfermagem 2006 novembro-dezembro; 14(6)

15. Okabe I, Fonseca RMGS. Violência contra a mulher: contribuições e limitações do sistema de informação. Rev Esc Enferm USP 2009; 43(2): 453-8

16. Gomes NP, Diniz NMF, Araújo AJS, Coelho TMF. Compreendendo a violência doméstica a partir das categorias gênero e geração. Acta Paul Enferm 2007; 20(4): 504-8. 
17. Lettiere A, Nakano MAS, Rodrigues DT. Violência contra a mulher: a visibilidade do problema para um grupo de profissionais de saúde. Rev Esc Enferm USP 2008; 42(3): 46773.

18. Araújo LM, Progianti JM, Vargens OMC. A Consulta de Enfermagem Ginecológica e a redução da violência de gênero. Rev Enferm UERJ 2004; 12:328-31.

19. Guedes RN, Silva ATMC, Coelho EAC. Violência conjugal: problematizando a opressão das mulheres vitimizadas sob olhar de gênero. Rev Eletr Enf. 2007 mai-ago; 9(2): 362-378.

20. Monteiro CFS, Souza IEO. Vivência da violência conjugal: fatos do cotidiano. Texto

Contexto Enferm. 2007 jan-mar; 16(1): 26-31 